A Decent Meal 
This page intentionally left blank 


\section{A Decent Meal}

B UILDING EMPATHY IN A

D IVIDE D A E RICA

$6 \rightarrow$

Michael Carolan

Redwood Press

STANFORD, CALIFORNIA 
(C) 2021 by the Board of Trustees of the Leland Stanford Junior University. All rights reserved.

No part of this book may be reproduced or transmitted in any form or by any means, electronic or mechanical, including photocopying and recording, or in any information storage or retrieval system without the prior written permission of Stanford University Press.

Printed in the United States of America on acid-free, archival-quality paper Library of Congress Cataloging-in-Publication Data

Names: Carolan, Michael S., author.

Title: A decent meal : building empathy in a divided America / Michael Carolan.

Description: Stanford, California : Redwood Press, 2021. | Includes bibliographical references and index.

Identifiers: LCCN 2021018472 (print) | LCCN 2021018473 (ebook) | ISBN 9781503613287 (cloth) | ISBN 9781503629547 (epub)

Subjects: LCSH: Empathy_Social aspects_United States. | Attitude change-United States-Experiments. | Social groups-United States-Psychological aspects. | Right and left (Political science) United States. | Attitude (Psychology) —United States. | Social psychology_United States. | Public opinion-United States.

Classification: LCC BF575.E55 C 3572021 (print) | LCC BF575.E55 (ebook) | DDC 152.4/1--dc23

LC record available at https://lccn.loc.gov/2021018472

LC ebook record available at https://lccn.loc.gov/2021018473

Cover design: Michel Vrana

Cover image: iStock (composite)

Text design: Kevin Barrett Kane

Typeset at Stanford University Press in 11/15 Arno Pro 